9. Tong XC, Weng SS, Xue F, Wu X, Xu TM, Zhang WH. First human infection by a novel avian influenza $\mathrm{A}(\mathrm{H} 7 \mathrm{~N} 4)$ virus. J Infect. 2018;77:249-57. https://doi.org/10.1016/j.jinf.2018.06.002

10. World Health Organization. Human infection with avian influenza A(H7N4) virus - China. Disease Outbreak News (DON) 2018 [cited 2018 Dec 17]. https://www.who.int/csr/don/22-february2018-ah7n4-china $<$ /eref $>$

Address for Correspondence: Erik A. Karlsson, Institut Pasteur du Cambodge Virology Unit, 5 Monivong Blvd, PO Box 983, Phnom Penh, Cambodia; email: ekarlsson@pasteur-kh.org

\title{
Mycobacterium marseillense Infection in Human Skin, China, 2018
}

\section{Bibo Xie, ${ }^{1}$ Yanqing Chen, ${ }^{1}$ Jian Wang, Wei Gao, Haiqing Jiang, Jiya Sun, Xindong Jin, Xudong Sang, Xiaobing Yu, Hongsheng Wang}

Author affiliations: Institute of Dermatology, Chinese Academy of Medical Sciences and Peking Union Medical College, Nanjing, China (B. Xie, Y. Chen, W. Gao, H. Jiang, H. Wang); Zhejiang Institute of Dermatology, Deqing, China (B. Xie, J. Wang, X. Jin, X. Sang, X. Yu); Jiangsu Key Laboratory of Molecular Biology for Skin Diseases and STIs, Nanjing (Y. Chen, W. Gao, H. Jiang, H. Wang); Suzhou Institute of Systems Medicine, Chinese Academy of Medical Sciences, Suzhou, China (J. Sun); Centre for Global Health, Nanjing Medical University, Nanjing (H. Wang)

DOI: https://doi.org/10.3201/eid2510.190695

We describe a case of facial skin infection and sinusitis caused by Mycobacterium marseillense in an immunocompetent woman in China in 2018. The infection was cleared with clarithromycin, moxifloxacin, and amikacin. Antimicrobial drug treatments could not be predicted by genetic analyses; further genetic characterization would be required to do so.

$M$ ycobacterium marseillense is a member of the M. avium complex (1) that has caused infections with lymphatic or pulmonary involvement sporadically in humans (2-4). We report M. marseillense infection involving facial skin in an immunocompetent woman in eastern China.

${ }^{1}$ These authors contributed equally to this article.
In April 2018, a 59-year-old woman was referred to our institute (Institute of Dermatology, Chinese Academy of Medical Sciences and Peking Union Medical College, Nanjing, China) for a 4-year history of an erythematous plaque with ulceration located on the right cheek. The primary lesion was a small erythematic patch that gradually developed into an asymptomatic ulcerative plaque (i.e., the plaque had no heat, swelling, pain, or pruritus). She also reported occasional bloody, purulent nasal discharge over the course of 2 years. Two years before visiting our hospital, cutaneous tuberculosis was suspected, so she received treatment for tuberculosis (rifampin, isoniazid, ethambutol, pyrazinamide) for 10 months. No obvious improvement was observed with this treatment. Her medical history was otherwise unremarkable.

On physical examination, an infiltrated erythematous plaque with yellow scales and crusts on the right cheek was visible (Figure, panel A). Routine laboratory tests showed no remarkable findings. The results of autoantibody and HIV tests were negative, and immune subset cell counts were unremarkable. Histologic examination showed infiltration of a large number of lymphocytes, plasma cells, and neutrophils and some tissue cells in the dermis (Appendix Figure 1, https://wwwnc.cdc.gov/EID/article/25/10/190695-App1.pdf). Computed tomography scan of the paranasal sinuses showed bilateral maxillary, right ethmoid, and frontal sinusitis (Figure, panel C). Culture and PCR for mycobacteria in nasal discharge yielded negative findings.

After 3 weeks of skin tissue culture at $32^{\circ} \mathrm{C}$ in Löwenstein-Jensen medium, we observed smooth, yolk-yellow bacterial colonies (Appendix Figure 2). Ziehl-Neelsen staining confirmed the cultured organism was acid-fast bacilli. Sequence analysis indicated that the complete genetic sequence of $16 \mathrm{~S}$ rRNA was $99.0 \%$, hsp $65100 \%$, and $r p o B$ 99.8\% homologous with M. marseillense strain FLAC0026. Phylogenetic analysis of the 16S rRNA sequence showed the isolate clustered with $M$. chimaera and $M$. intracellulare (Figure, panel D). Although the 16S rRNA gene sequence of the isolate was $100 \%$ similar to $M$. intracellulare subsp. yongonense 05-1390, the sequence similarities to $h \operatorname{sp} 65$ and $r p o B$ were relatively low. Sequence analyses suggested $M$. marseillense infection.

Referring to the guidelines for pulmonary $M$. avium complex disease, we treated the patient with the antimicrobial drugs clarithromycin, rifampin, and ethambutol (5). Afterward, in vitro drug susceptibility testing showed the isolate was sensitive to clarithromycin, azithromycin, and amikacin; moderately sensitive to moxifloxacin; and resistant to ethambutol and rifampin. Therefore, 3 months after initiating treatment, we changed the regimen to clarithromycin, moxifloxacin, and amikacin, which she received for 2 months. The patient's skin lesions healed gradually, and nasal symptoms disappeared, but a scar and erythema 

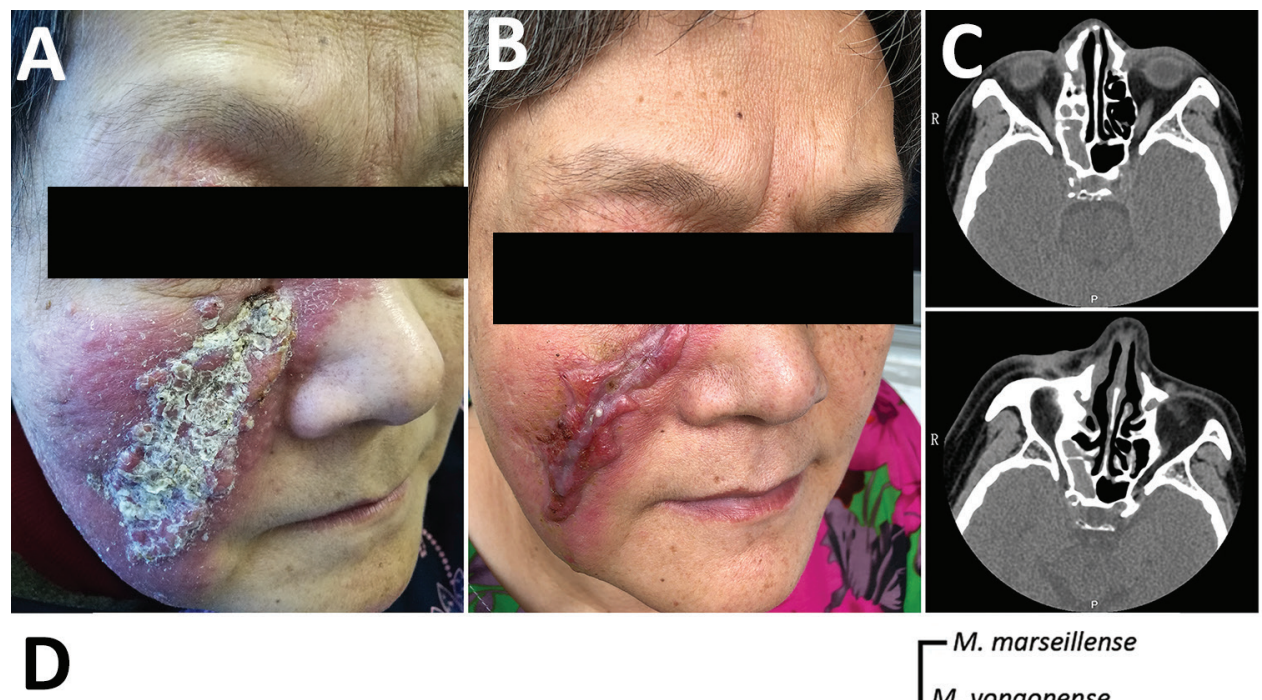

M. marseillense
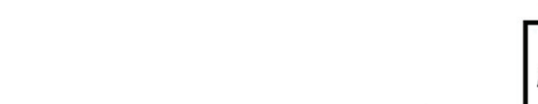

M. marseillense

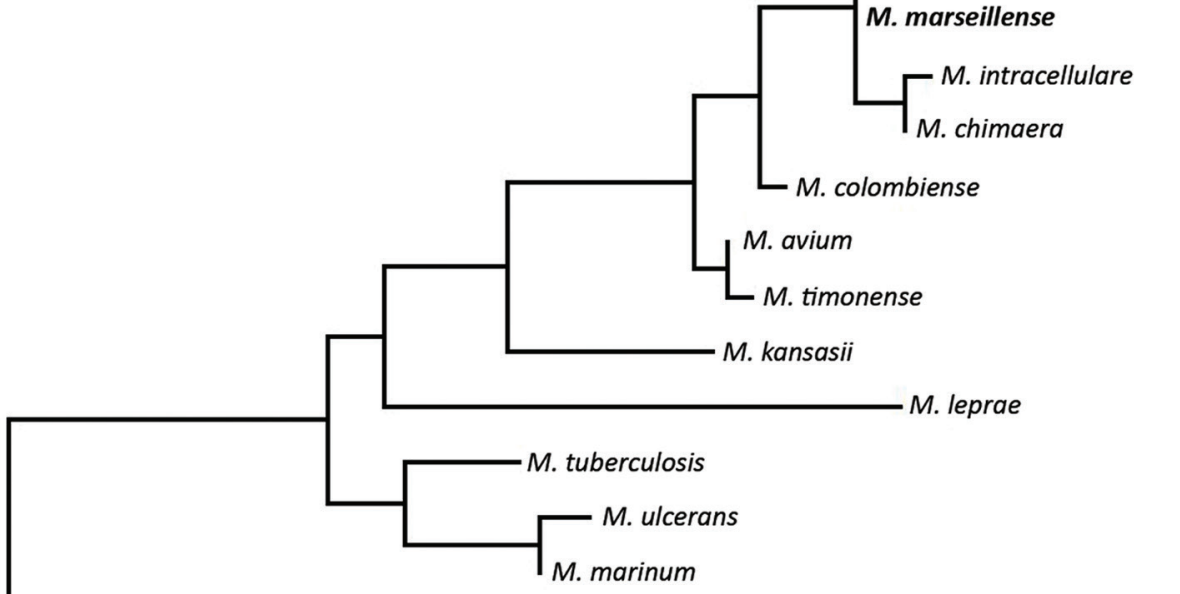

M. smegmatis
Figure. Skin lesions and computer tomography scans of woman with Mycobacterium marseillense skin infection, China, 2018, and genomic analysis of isolate. A, B) Facial skin lesion of woman with $M$. marseillense infection before and after treatment. Infiltrated erythematous plaque with yellowish scales and crusts (A) resolved to a scar after clearance of infection (B). C) Computed tomography imaging before treatment (top) shows heterogeneous hypersignal in right ethmoid sinus and after treatment (bottom) shows recovery of right ethmoid sinus. $P$, posterior; $R$, right. D) Phylogenetic tree constructed with 16S rRNA gene sequence of isolate from patient (bold) and other species. Scale bar indicates nucleotide substitutions per site.
0.0050

remained (Figure, panel B). Computed tomography scans of the paranasal sinuses showed the reduction of sinusitis (Figure, panel C). No recurrence was observed during 4 months of monitoring.

We characterized this isolate's genome (GenBank accession no. VASI0000000) further to help determine the cause of its virulence and resistance (Appendix Figure 3). Genetic analyses indicated the genome $(\approx 5,706,022 \mathrm{bp})$ contained 5,343 predicted genes, 3 rRNAs, and 48 tRNAs and had a GC content of $67.73 \%$. We annotated the genes functionally through multiple databases (Appendix Table 1, Figure 4). Using the Virulence Factors of Pathogenic Bacteria database, we identified 137 potential virulence genes (identity $>95.0 \%$, E value $<1 \times 10^{-5}$ ), such as type VII secretion system genes (e.g., esxH, esxC, esxH, and $\operatorname{esx} C)(6)$, in the isolate's genome (Appendix 2, https:// wwwnc.cdc.gov/EID/article/25/10/19-0695-App2.xlsx). In Comprehensive Antibiotic Resistance Database searches, we detected the antimicrobial drug resistance genes $m$ tr $A$, murA, and gyrA (identity $>90.0 \%$, E value $<1 \times 10^{-5}$; Appendix Table 2); $m$ tr $A$ modulates antimicrobial drug efflux, murA encodes the fosfomycin resistance protein, and gyrA encodes the fluoroquinolone resistance protein.

M. marseillense infections are rare in humans. Our case demonstrates that $M$. marseillense can cause infections in immunocompetent persons. For facial skin infection with M. marseillense, this and similar (7) reports indicate the need for vigilance of paranasal sinus infection. Although many potential virulence factors could be detected by genomic analysis, cases of infection and transmission with this bacterium are rarely reported, suggesting the presence of other influencing factors.

The drug resistance mechanisms of $M$. marseillense have not been completely elucidated. The drug susceptibility test results and treatment response we observed were generally consistent with those previously reported for 
cases of pulmonary infection, although sensitivity to rifampin and quinolones yielded various results (2-4). Drug susceptibility testing indicated that the isolate we obtained was resistant to ethambutol and rifampin. However, in genetic analyses, mutations associated with ethambutol and rifampin resistance were not detected. According to the Comprehensive Antibiotic Resistance Database, our isolate was resistant to fluoroquinolone, but drug susceptibility test results were inconsistent. Our results indicate that drug susceptibility testing should be performed for M. marseillense to guide antimicrobial drug treatment. If drug susceptibility results are absent, treatments including macrolides and amikacin appear to be reasonable.

\section{Acknowledgments}

We thank Jun Chen and Ying Shi for data analysis.

This manuscript was supported by grants from the Chinese Academy of Medical Science Innovation Fund for Medical Science (2017-I2M-B\&R-14, 2016-I2M-1-005), Jiangsu Provincial Science and Technology Project (BE2018619), National Science and Technology Major Project (2018ZX10101-001), Graduate Innovation Foundation of Peking Union Medical College (2018-1002-02-13), and Jiangsu Natural Science Foundation (BK20160377).

\section{About the Author}

Dr. Xie is a physician in the Department of Dermatology, Zhejiang Institute of Dermatology, Deqing, China, and formerly studied at the Institute of Dermatology, Chinese Academy of Medical Sciences and Peking Union Medical College, Nanjing, China. His major research interest is infectious diseases of the skin.

\section{References}

1. Ben Salah I, Cayrou C, Raoult D, Drancourt M. Mycobacterium marseillense sp. nov., Mycobacterium timonense sp. nov. and Mycobacterium bouchedurhonense sp. nov., members of the Mycobacterium avium complex. Int J Syst Evol Microbiol. 2009;59:2803-8. https://doi.org/10.1099/ijs.0.010637-0

2. Grottola A, Roversi P, Fabio A, Antenora F, Apice M, Tagliazucchi S, et al. Pulmonary disease caused by Mycobacterium marseillense, Italy. Emerg Infect Dis. 2014;20:1769-70. https://doi.org/10.3201/eid2010.140309

3. Kim SY, Yoo H, Jeong BH, Jeon K, Ha YE, Huh HJ, et al. First case of nontuberculous mycobacterial lung disease caused by Mycobacterium marseillense in a patient with systemic lupus erythematosus. Diagn Microbiol Infect Dis. 2014;79:355-7. https://doi.org/10.1016/j.diagmicrobio.2014.03.019

4. Azzali A, Montagnani C, Simonetti MT, Spinelli G, de Martino M, Galli L. First case of Mycobacterium marseillense lymphadenitis in a child. Ital J Pediatr. 2017;43:92. https://doi.org/10.1186/ s13052-017-0413-5

5. Griffith DE, Aksamit T, Brown-Elliott BA, Catanzaro A, Daley C, Gordin F, et al.; American Thoracic Society Mycobacterial Diseases Subcommittee; American Thoracic Society; Infectious Disease Society of America. An official ATS/IDSA statement: diagnosis, treatment, and prevention of nontuberculous mycobacterial diseases. (Erratum in: Am J Respir Crit Care Med 2007;175:744-5.) Am J Respir Crit Care Med. 2007;175:367-416. https://doi.org/10.1164/recm.200604-571ST

6. Abdallah AM, Gey van Pittius NC, DiGiuseppe Champion PA, Cox J, Luirink J, Vandenbroucke-Grauls CMJE, et al. Type VII secretion-mycobacteria show the way. Nat Rev Microbiol. 2007;5:883-91. https://doi.org/10.1038/nrmicro1773

7. Chen Y, Jiang J, Jiang H, Chen J, Wang X, Liu W, et al Mycobacterium gordonae in patient with facial ulcers, nosebleeds, and positive T-SPOT.TB test, China. Emerg Infect Dis. 2017;23:1204-6. https://doi.org/10.3201/eid2307.162033

Address for correspondence: Hongsheng Wang, Chinese Academy of Medical Sciences and Peking Union Medical College, Institute of Dermatology, St 12 Jiangwangmiao, Nanjing, Jiangsu, 210042, China; email:whs33@vip.sina.com

\section{Mary A.M. Rogers, Catherine Kim, Annika M. Hofstetter}

Author affiliations: University of Michigan, Ann Arbor, Michigan, USA (M.A.M. Rogers, C. Kim); University of Washington and Seattle Children's Research Institute, Seattle, Washington, USA (A.M. Hofstetter)

DOI: https://doi.org/10.3201/eid2510.190874

We evaluated rotavirus vaccination rates in the United States by using records from a nationwide health database. From data on 519,697 infants, we found $68.6 \%$ received the entire rotavirus vaccine series. We noted pockets of undervaccination in many states, particularly in the Northeast and in some western states.

$\mathrm{V}$ accination coverage in the United States frequently is evaluated with telephone and mailed surveys (1). However, telephone response rates have declined over the past 2 decades (2) and parents who choose not to vaccinate their children might be less likely to participate in surveys (3). 